\title{
LARGE ABELIAN SUBGROUPS OF SOME INFINITE GROUPS VANCE FABER
}

ABstract. A generalization of the following conjecture of W. R. Scott is proved. If $\left(H_{\alpha}\right)_{\alpha}$ is a well-ordered descending chain of subgroups of a group with the property that $H_{\beta}=\bigcap_{\alpha<\beta} H_{\alpha}$ for limit ordinals, then $\left[H_{0}: H_{\delta}\right] \leqq \Pi_{\alpha<\delta}\left[H_{\alpha}: H_{\alpha+1}\right]$. Using this, we show that the members of certain classes of infinite groups are guaranteed to have large abelian subgroups.

1. Introduction. Following Kurosh [5, p. 171], a totally ordered system $U$ of subgroups of a group is said to be complete if for an arbitrary subsystem of $\mathcal{U}$, the unions and the intersections of the subgroups forming the subsystem belong to $\mathcal{U}$. W. R. Scott [8, p. 21] has conjectured that, if $\left(H_{\alpha}\right)_{\alpha \leqq \delta}$ is a well-ordered descending complete system of subgroups of a group $H_{0}$, then

$$
\left[H_{0}: H_{\delta}\right] \leqq \prod_{\alpha<\delta}\left[H_{\alpha}: H_{\alpha+1}\right] .
$$

In a private communication, Scott has shown that this is indeed true for $\delta=\omega$, the first infinite ordinal, and has stated that under these same conditions both he and, independently, A. Kruse have proved that

$$
\left[H_{0}: H_{\delta}\right] \leqq\left[\prod_{\alpha<\delta}\left[H_{\alpha}: H_{\alpha+1}\right]\right]^{|\delta|} .
$$

In $\$ 3$ we shall establish a generalized form of Scott's conjecture from which the latter can be deduced. In addition, we shall find a lower bound for $\left[H_{0}: H_{\delta}\right]$ which will be useful in $\$ 4$.

If $m$ is a cardinal number, we define $\exp m=\exp ^{1} m=2^{m}$. Inductively, if $n$ is any positive integer, we define $\exp ^{n+1} m=\exp \exp ^{n} m$. In $\$ 4$ we utilize equation ( 1 ) to investigate the existence of large abelian subgroups of ccrtain infinite groups. For example, C. R. Kulatilaka has shown $\left[4\right.$, p. 241] that every infinite $S I^{*}$-group $G$

Received by the editors February 20, 1970 and, in revised form, August 20, 1970.

AMS 1969 subject classifications. Primary 2018, 2056, 2058; Secondary 2030, 2037.

Key words and phrases. Abelian subgroup, infinite group, well-ordered chain of subgroups, ordered system of subgroups, invariant series, FC-series, SI*-group, Fitting subgroup, Baer radical, Gruenberg radical, generalized continuum hypothesis, locally nilpotent, $F C$-nilpotent, ZA-group. 
has an infinite abelian subgroup $A$ such that $A \triangleleft^{2} G$ ( $A$ is normal in its normal closure, $\left.A^{G}\right)$. We shall show that every infinite $S I^{*}$-group $G$ has an abelian subgroup $A$ such that $\exp ^{2}|A| \geqq|G|$, in fact, Theorem 5 states that $G$ need only have an ascending invariant series with $F C$ factors. For similar results on large discrete subspaces of topological spaces, the reader should see [2] and [3].

2. Notation. Let $S$ and $T$ be sets. $S<T$ will always mean strict inclusion. The cardinality of $S$ will be denoted by $|S|$. If $G$ is a group and $H$ is a subgroup, we write $H \triangleleft^{n} G$ if there is an ascending normal series

$$
H=H_{0} \triangleleft H_{1} \triangleleft \cdots \triangleleft H_{n}=G
$$

from $H$ to $G$. $C(H)=C(H \leqq G)$ denotes the centralizer of $H$ in $G$, while $N(H)=N(H \leqq G)$ denotes the normalizer of $H$ in $G$. A transversal for $H$ in $G$ is a full set of distinct coset representatives for $H$ in $G$; if a transversal contains the identity, it is said to be normalized.

Let sym $|S|=\operatorname{sym}^{1}|S|$ be the order of the symmetric group on $|S|$ letters; if $n$ is a positive integer, define $\operatorname{sym}^{n+1}|S|=\operatorname{sym} \operatorname{sym}^{n}|S|$. We shall frequently make use of the fact that

$$
\mid \text { Aut }(G)|\leqq \operatorname{sym}| G \mid \text {, }
$$

where Aut $(G)$ is the group of automorphisms of $G$.

We assume the terminology of $\$ 57$ and $\$ 63$ of [5] to denote various classes of generalized solvable and nilpotent groups.

Let $F_{1}(G)$ be the set of all elements in $G$ which have at most a finite number of conjugates in G. As in [1], we define the upper $F C$-series of $G$ to be the ascending characteristic series

$$
E=F_{0}(G) \triangleleft F_{1}(G) \triangleleft \cdots \triangleleft F_{\alpha}(G) \triangleleft \cdots
$$

where $F_{\alpha+1}(G) / F_{\alpha}(G)=F_{1}\left(G / F_{\alpha}(G)\right)$, and if $\beta$ is a limit ordinal, then $F_{\beta}(G)=\bigcup_{\alpha<\beta} F_{\alpha}(G)$. If $F_{1}(G)=G, G$ is said to be an FC-group. If $F_{\alpha}(G)=G$ for some $\alpha$, then $G$ is a ZFC-group; if $\alpha$ is an integer, $G$ is FC-nilpotent.

If $m$ is an infinite cardinal, let $M_{1}(G)$ be the set of all elements in $G$ which have at most $m$ conjugates in $G$. By analogy with the upper $F C$-series, we define the upper $m C$-series of $G$ to be the ascending characteristic series

$$
E=M_{0}(G) \triangleleft M_{1}(G) \triangleleft \cdots \triangleleft M_{\alpha}(G) \triangleleft \cdots
$$

where $M_{\alpha+1}(G) / M_{\alpha}(G)=M_{1}\left(G / M_{\alpha}(G)\right)$, and if $\beta$ is a limit ordinal, then $M_{\beta}(G)=\bigcup_{\alpha<\beta} M_{\alpha}(G)$. If $M_{1}(G)=G, G$ is an $m C$-group; if $M_{\alpha}(G)=G$ for some $\alpha$, then $G$ is a $Z m C$-group. 
If $\mathfrak{X}$ is a class of groups, we let $\mathfrak{X} I^{*}$ be the class of all groups having an ascending invariant series with factors in $\mathfrak{X}$. If every subgroup and every homomorphic image of an $\mathfrak{X}$-group is an $\mathfrak{X}$-group, then the same holds true for $\mathfrak{X} I^{*}$-groups $[5, \S 56]$.

Let $U=\left(A_{\alpha}\right)_{\alpha \in W}$ be a complete ordered system of subgroups of a group $G$. We shall suppose that $W$ is ordered by a relation $<$ such that $\alpha<\beta$ implies that $A_{\alpha}<A_{\beta}$. If $\alpha$ has an immediate successor in $W$, we denote it by $\alpha+1$. Let $\mathcal{J}$ be the set of all $\alpha$ in $W$ for which $\alpha+1$ exists. If $W$ is well ordered, $\mathcal{U}$ is an ascending series; if $W$ with the inverse ordering is well ordered, $\mathcal{U}$ is a descending series.

If $\alpha$ is an ordinal, we say $\alpha$ is of the first kind if it has an immediate predecessor; otherwise, $\alpha$ is of the second kind. A limit ordinal is any nonzero ordinal of the second kind.

3. The index theorems.

THEOREM 1. Let $U=\left(A_{\alpha}\right)_{\alpha} \in w$ be a complete ordered system of subgroups of a group $G$ containing the whole group $G=A_{\mu}$ and some $A_{0}=\bigcap_{\alpha \in W} A_{\alpha}$. Then

$$
\left[G: A_{0}\right] \leqq \prod_{\alpha \in \mathcal{I}}\left[A_{\alpha+1}: A_{\alpha}\right]
$$

Proof. Let $R_{\alpha}$ be a transversal for $A_{\alpha}$ in $A_{\alpha+1}$ for each $\alpha \in \mathcal{J}$. Let $T_{\alpha}$ be a transversal for $A_{\alpha}$ in $G$ for each $\alpha \in W$. For each $g \in T_{0}$, let $g_{\alpha}$ be the unique element in $T_{\alpha}$ such that $A_{\alpha} g_{\alpha}=A_{\alpha} g$. If $\alpha \in \mathcal{J}$, then consider $A_{\alpha} g_{\alpha}\left(g_{\alpha+1}\right)^{-1}$. Since $A_{\alpha+1} g_{\alpha+1}=A_{\alpha+1} g$ and $A_{\alpha} g_{\alpha}=A_{\alpha} g$, it follows that $g\left(g_{\alpha+1}\right)^{-1} \in A_{\alpha+1}$ and that $g_{\alpha} g^{-1} \in A_{\alpha} \leqq A_{\alpha+1}$; and, consequently, that $g_{\alpha}\left(g_{\alpha+1}\right)^{-1} \in A_{\alpha+1}$. Thus we can define a unique point $F_{g}$ in the cartesian product, $\prod_{\alpha \in \mathcal{Z}} R_{\alpha}$, by the two conditions

(i) $F_{\mathrm{g}}(\alpha) \in R_{\alpha}$,

(ii) $A_{\alpha} F_{g}(\alpha)=A_{\alpha} g_{\alpha}\left(g_{\alpha+1}\right)^{-1}$

for all $\alpha \in \mathcal{2}$. If the function $F$ taking $g$ to $F_{g}$ is one-to-one, then $\left|T_{0}\right| \leqq \prod_{\alpha \in \mathcal{J}}\left|R_{\alpha}\right|$, the conclusion of the theorem.

If $F_{\mathrm{g}}=F_{h}$ for $g$ and $h$ in $T_{0}$, then by (ii)

$$
A_{\alpha} g_{\alpha}\left(g_{\alpha+1}\right)^{-1}=A_{\alpha} h_{\alpha}\left(h_{\alpha+1}\right)^{-1}
$$

for all $\alpha \in$ 2. Let $P(\alpha)$ be the statement that $g_{\alpha}=h_{\alpha}$ for all $\alpha \in W$. Since there is only one element in $T_{\mu}, P(\mu)$ obviously holds. If $[S(1), S(2)]$ is a Dedekind section taken in $W$ having the property that $P(\alpha)$ holds for all elements $\alpha \in S(2)$, then we can easily find some $\beta \in S(1)$ for which $P(\beta)$ holds. Suppose this were not the case, then $g_{\alpha} \neq h_{\alpha}$ for every $\alpha \in S(1)$. Since $A_{\alpha} g=A_{\alpha} g_{\alpha} \neq A_{\alpha} h_{\alpha}=A_{\alpha} h$, then $g^{-1} \notin A_{\alpha}$ for every $\alpha \in S(1)$; and so $g h^{-1} \notin \bigcup_{\alpha \in S(1)} A_{\alpha}$. But by 
assumption, $g h^{-1} \in A_{\alpha}$ for all $\alpha \in S(2)$, and thus $A_{\gamma}=\bigcup_{\alpha \in S(1)} A_{\alpha}$ must be the last element of $S(1)$. Similarly, $A_{\alpha} g=A_{\alpha} h$ for each $\alpha \in S(2)$ implies that $g h^{-1} \in \bigcap_{\alpha \in S(2)} A_{\alpha}$. Hence $\bigcap_{\alpha \in S(2)} A_{\alpha}$ cannot be in $S(1)$ and, therefore, must be the first element of $S(2)$, namely $A_{\gamma+1}$. But $\left(^{*}\right)$ and $g_{\gamma+1}=h_{\gamma+1}$ together yield $A_{\gamma} g_{\gamma}=A_{\gamma} h_{\gamma}$, contradicting the assumption that $g_{\gamma} \neq h_{\gamma}$.

Let $S^{\prime}(2)=\{\alpha \in W \mid \alpha \geqq \beta\}$ and let $S^{\prime}(1)$ be its complement in $W$. Then $\left[S^{\prime}(1), S^{\prime}(2)\right]$ is a section in $W$ with $S^{\prime}(2)>S(2)$. Since $g_{\beta}=h_{\beta}$, we have $g h^{-1} \in A_{\beta} \leqq A_{\alpha}$ for every $\alpha \geqq \beta$, that is, $g_{\alpha}=h_{\alpha}$ for all $\alpha \in S^{\prime}(2)$. Thus, by induction on the ordered set $W[9, \mathrm{p}$. 264], $P(\alpha)$ holds for all $\alpha \in W$.

To complete the proof, we note that $g_{\alpha}=h_{\alpha}$ for all $\alpha \in W$ implies that $g h^{-1} \in A_{\alpha}$ for all $\alpha$, and hence that $g h^{-1} \in \bigcap_{\alpha \in W} A_{\alpha}=A_{0}$. Thus $g=h$ and $F$ is one-to-one.

Statement (1) in the introduction is easily seen to be that special case of Theorem 1 in which $W$ with the inverse ordering is a wellordered set.

THEOREM 2. Let $u=\left(A_{\alpha}\right)_{\alpha \in W}$ be a complete ordered system of subgroups of a group $G$ containing the whole group $G=A_{\mu}$ and some $A_{0}=\bigcap_{\alpha \in W} A_{\alpha}$. Then

$$
\sum_{\alpha \in \mathcal{J}}\left[A_{\alpha+1}: A_{\alpha}\right] \leqq\left[G: A_{0}\right] .
$$

Proof. If $\mathcal{J}$ is finite, one can easily establish by induction that

$$
\sum_{\alpha \in \mathcal{J}}\left[A_{\alpha+1}: A_{\alpha}\right] \leqq \prod_{\alpha \in \mathcal{J}}\left[A_{\alpha+1}: A_{\alpha}\right],
$$

the desired result in this case.

If $\mathcal{J}$ is infinite, we shall establish the somewhat stronger statement

$$
\sum_{\alpha \in \mathcal{J}}\left[A_{\alpha+1}: A_{0}\right] \leqq\left[G: A_{0}\right] \text {. }
$$

Let $R_{\alpha}$ be a normalized transversal for $A_{\alpha}$ in $A_{\alpha+1}$ for each $\alpha \in \mathcal{J}$. Let $L_{\alpha}$ be a normalized transversal for $A_{0}$ in $A_{\alpha}$ for each $\alpha \in \mathcal{J}$. The main steps in the proof consist of showing that:

(i) $L_{\alpha} R_{\alpha}$ is a transversal for $A_{0}$ in $A_{\alpha+1}$ for each $\alpha \in \mathcal{J}$;

(ii) $\{1\} \cup_{\alpha \in \mathcal{I}}\left(L_{\alpha} R_{\alpha} \backslash L_{\alpha}\right)$ is a transversal for $A_{0}$ in $G$, and this union is disjoint;

(iii) $\left|L_{\alpha} R_{\alpha}\right| \leqq 2\left|L_{\alpha} R_{\alpha} L_{\alpha}\right|$.

Since $\mathcal{J}$ is infinite, (iii) implies (2). Details are omitted.

REMARK. If $u$ is a complete ordered system as in the theorem, let

$$
\Sigma(\mathcal{U})=\sum_{\alpha \in \mathcal{J}}\left[A_{\alpha+1}: A_{\alpha}\right], \quad \Pi(\mathcal{U})=\prod_{\alpha \in \mathcal{J}}\left[A_{\alpha+1}: A_{\alpha}\right] .
$$


If the complete ordered system $\mathcal{U}^{\prime}$ is a refinement of $u$, then it is easily shown that

$$
\Sigma\left(u^{\prime}\right) \leqq \Sigma(u) \leqq\left[G: A_{0}\right] \leqq \Pi(u) \leqq \Pi\left(\mathcal{U}^{\prime}\right),
$$

that is, the finer the system becomes, the worse the approximation to $\left[G: A_{0}\right]$. However, using a general fact from cardinal arithmetic $[9$, p. 418], we obtain

$$
\Pi(u) \leqq[\Sigma(u)]^{|2|}
$$

By examining first the case where $\mathcal{J}$ is finite, and then the case where $\partial$ is infinite, one gets

$$
\Sigma(\mathcal{U}) \leqq\left[G: A_{0}\right] \leqq \Pi(\mathcal{U}) \leqq \exp \Sigma(\mathcal{U}) \leqq \exp \left[G: A_{0}\right]
$$

Hence both $\Sigma(\mathcal{\Sigma})$ and $\Pi(\mathcal{U})$ are limited in range. In fact, if $G$ is infinite and we assume the generalized continuum hypothesis, there are only two possible values for $\left[G: A_{0}\right]: \Sigma(\mathcal{L})$ or $\exp \Sigma(\mathcal{U})$; also, there are only two possible values for $\Pi(\mathcal{U}):\left[G: A_{0}\right]$ or $\exp \left[G: A_{0}\right]$. The only combination of these possibilities which cannot occur is $\left[G: A_{0}\right]=\exp \Sigma(\mathcal{L})$ and $\Pi(\mathcal{U})=\exp \left[G: A_{0}\right]$.

\section{Large abelian subgroups.}

LEMMA [4, p. 240]. If $A$ is a maximal normal abelian subgroup of a group $G$ and if $G / A$ is a ZA-group, then $A=C(A)$.

Conollary 1. If $A$ is a maximal normal abelian subgroup of an infinite group $G$ and if $G / A$ is a ZA-group, then $\exp |A| \geqq|G|$.

Proof [4, p. 240]. By the lemma, $A=C(A)$. Hence $G / A$ is isomorphic to a subgroup of the group of automorphisms of $A$; and so if $A$ were finite, $G$ would also be finite. Thus $A$ must be infinite and $\mid$ Aut $(A)|\leqq \exp | A \mid$. It follows that

$$
|G|=|A||G| A|\leqq| A|| \text { Aut }(A)|\leqq \exp | A \mid .
$$

THEOREM 3. If $A_{1}$ is a normal abelian subgroup of an infinite group $G$ and if $G / A_{1}$ is a ZFC-group, then $G$ has an abelian subgroup $A$ containing $A_{1}$ such that $\exp |A| \geqq|G|$.

Proof. Let $A_{0}=E, H_{0}=G$ and $H_{1}=C\left(A_{1} \leqq G\right)$. Inductively, suppose that we have defined the ascending chain $\left(A_{\alpha}\right)_{\alpha<\beta}$ and the descending chain $\left(H_{\alpha}\right)_{\alpha<\beta}$ such that $A_{\alpha} \leqq Z\left(H_{\alpha}\right)$ for all $\alpha<\beta$. If $\beta$ is a limit ordinal, let $A_{\beta}=\bigcup_{\alpha<\beta} A_{\alpha}$ and let $H_{\beta}=\bigcap_{\alpha<\beta} H_{\alpha}$. If $\beta=\alpha+1$ for some ordinal $\alpha$, let the superscript - denote homomorphic images in $H_{\alpha} / Z\left(H_{\alpha}\right)$. Since $\bar{H}_{\alpha}$ is a $Z F C$-group, if it is not trivial, there exists 
an element $x_{\alpha+1}$ in $H_{\alpha} \backslash Z\left(H_{\alpha}\right)$ such that $\bar{x}_{\alpha+1}$ is in $F_{1}\left(\bar{H}_{\alpha}\right)$. Let $A_{\alpha+1}$ $=\left\langle x_{\alpha+1}\right\rangle Z\left(H_{\alpha}\right)$, and let $H_{\alpha+1}=C\left(A_{\alpha+1} \leqq H_{\alpha}\right)$. Hence for all $\alpha$, $A_{\alpha}<A_{\alpha+1}$ unless $H_{\alpha}=Z\left(H_{\alpha}\right)$, that is, unless $H_{\alpha}$ is abelian. Let $\gamma$ be the first ordinal such that $H_{\gamma}$ is abelian.

We note that since $\bar{x}_{\alpha+1}$ has only a finite number of conjugates in $\bar{H}_{\alpha}, \quad n_{\alpha+1}=\left[H_{\alpha}: N\left(A_{\alpha+1} \leqq H_{\alpha}\right)\right]=\left[\bar{H}_{\alpha}: N\left(\bar{A}_{\alpha+1} \leqq \bar{H}_{\alpha}\right)\right]$ is finite. Thus

$$
\begin{aligned}
{\left[H_{\alpha}: H_{\alpha+1}\right] } & =n_{\alpha+1}\left[N\left(A_{\alpha+1} \leqq H_{\alpha}\right): C\left(A_{\alpha+1} \leqq H_{\alpha}\right)\right] \\
& \leqq n_{\alpha+1} \mid \text { Aut }\left(A_{\alpha+1}\right) \mid .
\end{aligned}
$$

Hence by Theorem 1

$$
\begin{aligned}
|G|=\left|H_{\gamma}\right|\left[G: H_{\gamma}\right] & \leqq\left|H_{\gamma}\right| \prod_{\alpha<\gamma}\left[H_{\alpha}: H_{\alpha+1}\right] \\
& \leqq\left|H_{\gamma}\right| \prod_{\alpha<\gamma} n_{\alpha+1} \mid \text { Aut }\left(A_{\alpha+1}\right) \mid
\end{aligned}
$$

and so if $\dot{H}_{\gamma}$ were finite, $G$ would also be finite. Thus $H_{\gamma}$ is infinite and it follows by equation (2) that

$$
\begin{aligned}
&|G| \leqq\left|H_{\gamma}\right| \prod_{\alpha<\gamma} \boldsymbol{\aleph}_{0}{ }^{\left|A_{\alpha+1}\right|}=\left|H_{\gamma}\right| \boldsymbol{\aleph}_{0}{ }^{\Sigma_{\alpha<\gamma}\left|A_{\alpha+1}\right|} \\
& \leqq\left|H_{\gamma}\right| \boldsymbol{\aleph}_{0}\left|H_{\gamma}\right|=\exp \left|H_{\gamma}\right| .
\end{aligned}
$$

THeOREM 4. If $A_{1}$ is a normal FC-subgroup of an infinite FCI*group $G$, then $G$ has a ZFC-subgroup $H$ containing $A_{1}$ such that $\exp |H| \geqq|G|$.

Proof. Let $H_{0}=G$ and let $A_{0}=E$. If possible, let $A_{\alpha+1}$ be a normal subgroup of $H_{\alpha}$ such that $A_{\alpha+1} / A_{\alpha}$ is a nontrivial normal $F C$ subgroup of $H_{\alpha} / A_{\alpha}$, then let $H_{\alpha+1}$ be the normal subgroup of $H_{\alpha}$ such that $H_{\alpha+1} / A_{\alpha}=\left(A_{\alpha+1} / A_{\alpha}\right) C\left(A_{\alpha+1} / A_{\alpha} \leqq H_{\alpha} / A_{\alpha}\right)$. If $\beta$ is a limit ordinal, let $A_{\beta}=\bigcup_{\alpha<\beta} A_{\alpha}$ and let $H_{\beta}=\bigcap_{\alpha<\beta} H_{\alpha}$. Since $H_{\alpha} / A_{\alpha}$ is an FCI group, if it is not trivial, then it has a nontrivial normal $F C$-subgroup. Hence $A_{\alpha+1}>A_{\alpha}$ unless $H_{\alpha}=A_{\alpha}$. Thus there is a first ordinal $\gamma$ such that $H_{\gamma}=A_{\gamma}$.

Since $A_{\alpha+1} / A_{\alpha}$ is an $F C$-group, using the definition of $H_{\alpha+1}$, we see that each element in $A_{\alpha+1} / A_{\alpha}$ has only a finite number of conjugates in $A_{\gamma} / A_{\alpha}$. Thus $A_{\gamma}$ is a $Z F C$-group. It follows by the method of the previous argument (Theorem 3 ) that

$$
\left[G: A_{\gamma}\right] \leqq \prod_{\alpha<\gamma}\left[H_{\alpha} / A_{\alpha}: H_{\alpha+1} / A_{\alpha}\right] \leqq \prod_{\alpha<\gamma}\left|\operatorname{Aut}\left(A_{\alpha+1} / A_{\alpha}\right)\right| .
$$

Again, $A_{\gamma}$ must be infinite, so by Theorem 2 


$$
\left[G: A_{\gamma}\right] \leqq \prod_{\alpha<\gamma} \boldsymbol{K}_{0}\left|A_{\alpha+1} / A_{\alpha}\right| \leqq \boldsymbol{K}_{0} \Sigma_{\alpha<\gamma}\left|A_{\alpha+1}\right| A_{\alpha}\left|\leqq \boldsymbol{K}_{0}\right| A_{\gamma} \mid
$$

Thus

$$
|G| \leqq \boldsymbol{\aleph}_{0}\left|A_{\gamma}\right|=\exp \left|A_{\gamma}\right|
$$

Theorem 3 together with Theorem 4 gives

THEOREM 5. If $G$ is an infinite $F C I^{*}$-group and if $A_{1}$ is a normal abelian subgroup of $G$, then $G$ has an abelian subgroup $A$ containing $A_{1}$ such that $\exp ^{2}|A| \geqq|G|$.

We would like to show that an infinite group $G$, every subgroup of which is subnormal, has an abelian subgroup $A$ such that $\exp ^{2}|A| \geqq|G|$. Since $S I^{*}$-groups are $\mathrm{FCI}^{*}$-groups, this would certainly be the case if $G$ were always an $\mathrm{SI}^{*}$-group; but this is an open problem, so we settle for

THEOREM 6. If an infinite group $G$ has an abelian subgroup $A$ such that $A \triangleleft^{n} G$ and such that $|C(A)| \leqq \operatorname{sym}|A|$, then $\exp ^{n}|A| \geqq|G|$.

Proof. Let $A=A_{0} \triangleleft A_{1} \triangleleft \cdots \triangleleft A_{n}=G$ be a finite normal series from $A$ to $G$. For each integer $k<m$,

$$
\begin{aligned}
\left|A_{k+1}\right| & \leqq\left|N\left(A_{k}\right)\right|=\left[N\left(A_{k}\right): C\left(A_{k}\right)\right]\left|C\left(A_{k}\right)\right| \\
& \leqq \operatorname{sym}\left|A_{k}\right| \cdot|C(A)| \leqq \operatorname{sym}\left|A_{k}\right| .
\end{aligned}
$$

Proceeding by induction, we get

$$
|G|=\left|A_{n}\right| \leqq \operatorname{sym}^{n}|A| .
$$

Again, $A$ cannot be finite, so $\operatorname{sym}^{n}|A|=\exp ^{n}|A|$.

THeOREM 7. If $H_{1}$ is a normal $m C$-subgroup of an infinite $m C I^{*}$ -

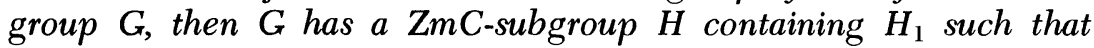
$\exp |H| \geqq|G|$. If $A_{1}$ is a normal abelian subgroup of an infinite $\mathrm{ZmC}$-group $H$, then $H$ has an abelian subgroup A containing $A_{1}$ such that $m^{|A|} \geqq|H|$. Thus, if $A_{1}$ is a normal abelian subgroup of an infinite $m C I^{*}$-group $G$, then $G$ has an abelian subgroup A containing $A_{1}$ such that $\exp m^{|A|} \geqq|G|$.

Proof. The arguments used above are easily applied here. Details are omitted.

Remark. If $G$ is any group, the Fitting subgroup of $G, \nu(G)$, is defined to be the product of all of the normal nilpotent subgroups of $G$. If $G$ coincides with $\nu(G), G$ is said to be a Fitting group. If $G$ is an $S I^{*}$-group, then $C(\nu(G))$ is contained in $\nu(G)$. (For details see $\left[7\right.$, p. 16].) Thus, if $G$ is an infinite $S I^{*}$-group, $\exp |\nu(G)| \geqq|G|$. 
Consequently, if we knew that every infinite Fitting group $N$ had an abelian subgroup $A$ such that $\exp |A| \geqq|N|$, then we could easily deduce that every infinite $S I^{*}$-group $G$ has an abelian subgroup $A$ with $\exp ^{2}|A| \geqq|G|$. We show

THEOREM 8. If one assumes the generalized continuum hypothesis, then every infinite Fitting group $G$ has an abelian subgroup A such that $\exp |A| \geqq|G|$.

Proof. Since Fitting groups are $S I^{*}$-groups, by Theorem $5, G$ has an abelian subgroup $A$ with $\exp ^{2}|A| \geqq|G|$.

Let $\boldsymbol{\kappa}_{\alpha}$ be the order of $G$. If $\boldsymbol{\alpha}$ is of the second kind, then we must have $|A|=|G|$. If $\alpha$ is of the first kind, but $\alpha-1$ is of the second kind, then we cannot have $\exp |A|<|G|$. For, if $|A|<\boldsymbol{\aleph}_{\alpha-1}$, then $\exp ^{2}|A|<\boldsymbol{\aleph}_{\alpha-1}$, while if $|A|=\boldsymbol{\aleph}_{\alpha-1}$, then $\exp |A|=\boldsymbol{\aleph}_{\alpha}=|G|$.

So we may assume that neither $\alpha$ nor $\alpha-1$ is of the second kind. If $\left|x^{G}\right|=|G|$ for some $x \in G$, then by Corollary $1, x^{G}$ has an abelian subgroup $B$ with $\exp |B| \geqq\left|x^{G}\right|=|G|$. Thus we may assume that $\left|x^{G}\right|<|G|$ for all $x$ in $G$. But then $G$ is an $\boldsymbol{\aleph}_{\alpha-1} C$-group; and so by Theorem $7, G$ has an abelian subgroup $B$ such that $\boldsymbol{\aleph}_{\alpha-1}^{|B|} \geqq \boldsymbol{\aleph}_{\alpha}$. If $|B| \leqq \boldsymbol{\aleph}_{\alpha-2}$, then $\boldsymbol{\aleph}_{\alpha-1}^{|B|} \leqq \boldsymbol{\aleph}_{\alpha-1}^{\boldsymbol{K}_{\alpha-2}}=\boldsymbol{\aleph}_{\alpha-1}$, a contradiction. Thus $|B| \geqq \boldsymbol{\aleph}_{\alpha-1}$; but then $\exp |B| \geqq \exp \boldsymbol{\aleph}_{\alpha-1}=\boldsymbol{\aleph}_{\alpha}$.

REMARK. If $G$ is any group, the Gruenberg (respectively, Baer) radical of $G, \rho(G)$, is the group generated by all the ascendant (respectively, subnormal) abelian subgroups of $G[7$, p. 100]. One can prove that if $G$ is an $S N^{*}$-group (respectively, $S J^{*}$-group), then $C(\rho(G))$ is contained in $\rho(G)$. (See, for example, [6, p. 352].) Thus, if $G$ is an infinite $S N^{*}$-group (respectively, $\mathrm{SJ}^{*}$-group), then $\exp |\rho(G)| \geqq|G|$. We note, however, that the Kovacs-Neumann example $[7, \mathrm{p} .110]$ is an infinite locally nilpotent $p$-group with trivial Gruenberg radical. If we generalize this example by wreathing together $\omega_{\omega}$ copies (instead of only $\omega_{1}$ copies) of $Z_{p}$, and if we assume the generalized continuum hypothesis, we then discover that there exists a locally finite p-group $G$ in which each abelian subgroup $A$ has $\exp ^{n}|A|<|G|$ for all positive integers $n$. For details, see [7, p. 111].

There is an infinite two-step nilpotent group $G$ which has a maximal normal abelian subgroup $A$ such that $\exp |A|=|G|[8,9.2 .17]$. This shows that the bounds given in Corollary 1 and Theorem 3 are the best possible. The author does not know whether any of the other results are the best possible. There seems to be no known counterexample to the following question: Does every locally nilpotent group of order $>\exp m$ have an abelian subgroup of order $>m$ ? 
Acknowledgement. This paper contains many of the results presented by the author to the Graduate School of Arts and Sciences of Washington University in partial fulfillment of the requirements for the Ph.D. degree. During the years 1968-1970, the author was supported by an NDEA Title IV Fellowship. The author wishes to express his gratitude both to Professor W. R. Scott for several enlightening communications, and to Professor F. Haimo, his advisor, whose great patience and optimistic encouragement helped to make this paper possible.

\section{BiBLIOGRAPHY}

1. F. Haimo, The FC-chain of a group, Canad. J. Math. 5 (1953), 498-511. MR 16, 216.

2. A. Hajnal and I. Juhász, Discrete subspaces of topological spaces, Nederl. Akad. Wetensch. Proc. Ser. A 70 = Indag. Math. 29 (1967), 343-356. MR 37 \#4769.

3. — Discrete subspaces of topological spaces. II, Nederl. Akad. Wetensch. Proc. Ser. A 72 = Indag. Math. 31 (1969), 18-30. MR 41 \#9177.

4. C. R. Kulatilaka, Infinite Abelian subgroups of some infinite groups, J. London Math. Soc. 39 (1964), 240-244. MR 28 \#5112.

5. A. G. Kuroš, The theory of groups. Vol. II, GITTL, Moscow, 1953; English transl., Chelsea, New York, 1960. MR 15, 501; MR 22 \#727.

6. R. E. Phillips and C. R. Combrink, A note on subsolvable groups, Math. Z. 92 (1966), 349-352. MR 33 \#5732.

7. D. J. S. Robinson, Infinite soluble and nilpotent groups, Queen Mary College Math. Notes, University of London, London, England, 1968.

8. W. R. Scott, Group theory, Prentice-Hall, Englewood Cliffs, N. J., 1964. MR 29 \#4785.

9. W. Sierpiński, Cardinal and ordinal numbers, 2nd rev. ed., Monografie Mat., vol. 34, PWN, Warsaw, 1965. MR 33 \#2549.

WAShington University, ST. Louis, Missouri 63130 


$$
\text { . }
$$

